\title{
Finite Element Assisted Method of Estimating Equivalent Circuit Parameters for a Superconducting Synchronous Generator With a Coreless Rotor
}

\author{
Bartosz Lukasik, Kevin F. Goddard, and Jan K. Sykulski \\ School of Electronics and Computer Science, University of Southampton, SO17 1BJ, UK
}

\begin{abstract}
The paper outlines methods developed to obtain circuit parameters of a superconducting synchronous generator with a coreless rotor. The need for full three-dmensional (3D) finite element modeling is emphasized and appropriate techniques devised to estimate relevant equivalent characteristics. The methods described use steady-state ac models, predominantly in the rotor frame of reference; the use of transient or full rotating machine models is avoided.
\end{abstract}

Index Terms-Coreless rotor, equivalent circuit parameters, superconducting synchronous generator.

\section{INTRODUCTION}

$\mathbf{R}$ ECENT advances in high-temperature superconducting (HTS) materials have opened opportunities to consider new generation of electrical power devices. However, a successful prediction of circuit parameters for such novel machines can pose significant difficulties. Classical techniques are not always applicable to superconducting machines because of a different construction. This paper reports on some of the challenges faced during the process of circuit parameters estimation for a superconducting synchronous generator with the coreless rotor designed at the University of Southampton.

\section{HTS CORELESS ROTOR DESIGN}

The rotor is of a coreless type, i.e., coils are not wound around an iron core and instead a nonmagnetic material is used. This approach poses several significant difficulties; for example, the lack of a magnetic core increases the magnetic field in the superconductor; to alleviate this problem, the rotor is designed as a stack of coils and flux diverters (Fig. 1) made of 9\% nickel steal, a material suitable for low temperatures and of high saturation flux density. The rotor is placed in a $0.9 \mathrm{~mm}$ thick copper tube which acts as a shield against higher order harmonics induced by the stator winding [1].

It has been shown through simulations that the magnetic characteristics of the above design are very satisfactory. Unfortunately though, producing an acceptable mechanical design for this arrangement proved very difficult, in particular when thermal contraction was taken into account. Consequently, the design of the rotor now under construction at Southampton differs significantly from that considered here; notwithstanding, the methods developed for equivalent circuit parameters estimation are still applicable.

\section{STATOR}

The stator was taken from a conventional $100 \mathrm{kVA}$, two-pole induction machine, with the bore diameter of $330 \mathrm{~mm}$ and iron

Manuscript received October 07, 2008. Current version published February 19, 2009. Corresponding author: B. Lukasik (e-mail: bl05r@ecs.soton.ac.uk).

Color versions of one or more of the figures in this paper are available online at http://ieeexplore.ieee.org.

Digital Object Identifier 10.1109/TMAG.2009.2012572

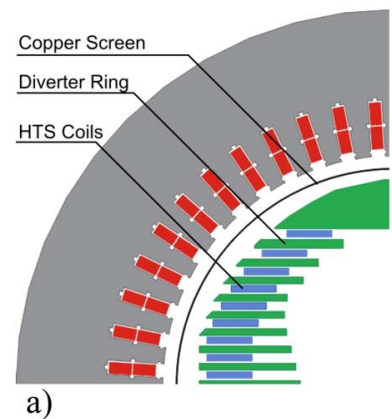

b)

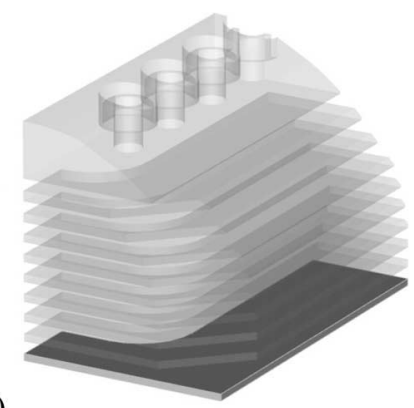

Fig. 1. Initial HTS rotor design. Stack of coils, diverters, and the copper screen are shown on the left, while a 3D view of the rotor is on the right.

length of $325 \mathrm{~mm}$. A short-pitched (14/24) two-layer winding with three-turn coils is distributed in 48 slots and connected in a parallel star arrangement. Using an existing stator introduced additional constraints on the design, in particular regarding the control of higher harmonics in the voltage waveform.

\section{THE NEED FOR 3D MODELING}

Modeling methods employed by most designers are based on a two-dimensional (2D) representation of the machine [2]. The parameters are obtained straight from the magnetic field solution of an FE model, or by coupling it with an external circuit to monitor voltage and current outputs and thus obtain the parameters by simulating one of the classical tests as performed on a real machine. All these techniques are based on a well-established general machine theory aided by a number of simplifications which make it possible to use a 2D model. The true three-dimensional (3D) nature of the field is then accommodated by introducing extra parameters, such as the end winding reactance. The approach is often accurate enough for machines of classical construction with iron cores. The majority of synchronous machines have a damper winding with well-defined current paths, which can be modeled in a similar manner. Unfortunately, the 2D simplification has proven inappropriate for the HTS coreless generator.

In addition to the low reactances of the central 2D section of the machine caused by the large air gap and the absence of a rotor core, which increases the relative importance of the endwinding inductances, additional flux paths around the ends of 
the flux diverter rings complicate the analysis. These flux paths allow flux to pass from one side of the rotor to the other without flowing through the central space, increasing the quadrature reactances and making the field in the central section of the machine more 3D. It was therefore decided that 3D finite-element models should be used for everything except preliminary design investigations.

A further difficulty arises when analyzing eddy currents in the copper screen, since this is a continuous sheet that does not force the current to follow well-defined paths. Using an arbitrary set of mode shapes to define the current distribution in the copper screen due to stator MMF harmonics would be difficult. Consequently, the screen is not treated as a winding; instead, its conductivity is included in the finite-element models as a material property.

\section{Modeling In the Rotor Frame of ReFERENCE}

Although, in principle, it is now possible to build and solve a full 3D transient rotating machine model of a generator, such models are computationally very expensive. Moreover, models that include circuit windings with complex geometry usually failed to mesh. Since rotating machine models require these circuit windings to represent currents that flow in the external circuits, the use of such models for design purposes cannot yet be considered practical with available software.

Instead, the generator is modeled using a series of steady-state ac models, predominantly in the rotor frame of reference. The method is related to the well-known method of estimating the reactances and time constants of a synchronous machine from the results of standstill frequency response tests [3].

This test is based on an assumption that by finding a number of transfer functions about some operating point, describing the electrical responses of stator and rotor quantities, parameters may be found by fitting the obtained data to the assumed model of the proper order. The transfer functions are described by the following equations:

$$
\begin{aligned}
& \Delta \Psi_{d}(s)=G(s) \Delta e_{f d}(s)-L_{d} \Delta i_{d}(s) \\
& \Delta \Psi_{q}(s)=-L_{q}(s) \Delta i_{q}(s)
\end{aligned}
$$

where $\Psi_{d}$ and $\Psi_{q}$ are direct and quadrature axis flux linkages, $i_{d}$ and $i_{q}$ are corresponding stator currents, $e_{f d}$ is the machine field voltage at a particular operating point, and $\Delta$ means a small perturbation, $L_{d}(s)$ - the direct axis operational inductance-is the Laplace transform of the ratio of direct axis armature flux linkages to the direct axis current with the field winding shortcircuited, $L_{q}(s)$ is analogous in quadrature axis, and $G(s)$ - the armature to field transfer function-is the Laplace transform of the ratio of the direct axis armature flux linkages to the field voltage with the armature open-circuited.

The results are then fitted into the function of the proper order [4] defined as

$$
\begin{aligned}
& x_{d}(s)=x_{d} \frac{\left(1+s T_{d}^{\prime}\right)\left(1+s T_{d}^{\prime \prime}\right) \ldots}{\left(1+s T_{d 0}^{\prime}\right)\left(1+s T_{d 0}^{\prime \prime}\right) \ldots} \\
& x_{q}(s)=x_{q} \frac{\left(1+s T_{q}^{\prime}\right) \ldots}{\left(1+s T_{q 0}^{\prime}\right) \ldots}
\end{aligned}
$$

where $T_{d}^{\prime}, T_{q}^{\prime}, T_{d}^{\prime \prime} \ldots$ are appropriate time constants.

It is apparent that some errors are inherent in the use of such test results to predict the behavior of a machine that is rotating. These errors arise because the field in the test only has one frequency, whereas the equivalent circuit is intended to represent a situation in which the field on the rotor has a number of different frequencies; the main field is at a low frequency, the field due to stator MMF harmonics differs from this by a multiple of six times the supply frequency, and stator slots modulate the field at multiples of the stator slot passing frequency.

For the machine considered, the errors introduced by using the wrong frequency for the field driven by space harmonics of the stator MMF are likely to be particularly large. There are two reasons for this: first, because the stator winding has a very short coil pitch and so has larger MMF harmonics, and second, because the damper winding is a copper sheet in the air gap rather than a set of bars buried in slots.

In a real test, there is little that can be done about these errors other than to make some estimates of their effect and make appropriate adjustments to the data. However, if the test results are replaced by the results of finite-element modeling, it is possible to model the field of the stator MMF harmonics separately at one or more appropriate frequencies, and combine the results by superposition. The only stator currents that need to be included in the low-frequency models are the direct-axis and the quadrature-axis sinusoidal components.

The use of superposition implies the use of a linear model; the stator core is therefore modeled as a linear anisotropic material. However, in the rotor iron (flux diverters), the rotor screen ensures that the high-frequency flux components driven by harmonics of the stator MMF are small; hence, we may neglect these high-frequency fields in a nonlinear model of the rotor. Other restrictions on the use of nonlinearity in the low-frequency model of the rotor will be explained later.

In addition to neglecting the effects of the high-frequency fields on the permeability of the flux diverters, the effects of flux diverters on the high-frequency fields are also ignored. The models that are used to predict the effects of the stator MMF harmonics on the stator flux linkage therefore have no flux diverters; the rotor is represented by the copper screen alone. This simplification greatly increases the symmetry of these models. It is therefore possible to employ superposition again to predict the field of the stator MMF harmonics from that of a single pair of coils. This reduction in the number of coils in the model greatly reduces the computational effort expended to solve the finite-element models.

To estimate the inductive energy due to harmonics of the stator MMF, the model is first post-processed to determine the flux linking each coil due to the current imposed in the one pair of coils that is included in the model. The flux linkages are calculated by integrating the normal component of $\mathrm{B}$ over a series of quadrilateral patches, the edges of which define six current paths uniformly distributed over the cross section of each three-turn coil. The flux linkage data is scaled to form a mutual inductance vector, and expanded to form a complete mutual inductance matrix (Fig. 2). Using this matrix, the inductive energy can be found for any distribution of stator currents by matrix multiplication. The simplest estimate of the energy due to the MMF harmonics 


$$
\left[\begin{array}{c}
L_{1,1} \\
M_{1,2} \\
M_{1,3} \\
\vdots \\
M_{1, n}
\end{array}\right] \Rightarrow\left[\begin{array}{ccccc}
L_{1,1} & -M_{1, n} & -M_{1, n-1} & \cdots & -M_{1,2} \\
M_{1,2} & L_{1,1} & -M_{1, n} & \cdots & -M_{1,3} \\
M_{1,3} & M_{1,2} & L_{1,1} & \cdots & -M_{1,4} \\
\vdots & \vdots & \vdots & \ddots & \vdots \\
M_{1, n} & M_{1, n-1} & M_{1, n-2} & \cdots & L_{1,1}
\end{array}\right]
$$

DIRECT AND QUADRATURE REACTANCES OBTAINED FROM 2D AND 3D SIMULATIONS

\begin{tabular}{|c|c|c|}
\hline Parameter & Result from 2D & Results from 3D \\
\hline$X_{d}$ [p.u.] & 0.1 & 0.12 \\
\hline$X_{q}$ [p.u.] & 0.15 & 0.18 \\
\hline
\end{tabular}

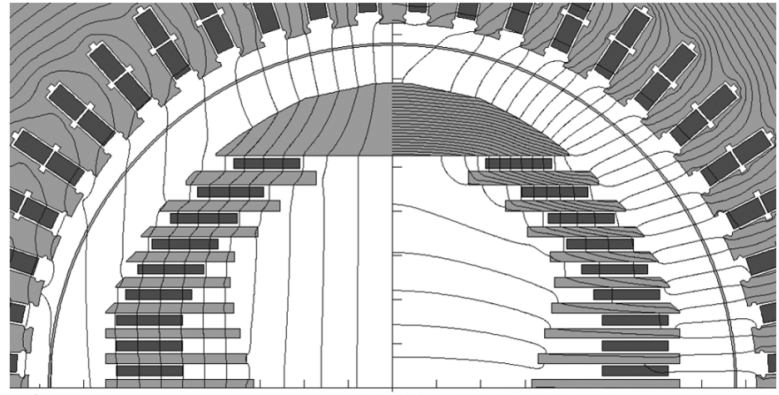

a)

b) range of frequencies involved this variation is small. Since most of the additional energy is associated with rotor currents at six times the output frequency of the generator, the errors are negligible if the model is solved at this frequency.

\section{TREATMENT OF NONLINEARITY}

As noted above, the use of a separate high-frequency model to predict the field due to harmonics of the stator MMF does not preclude the use of nonlinear material properties in the rotor of the low-frequency model. However, since the 3D software does not produce small-signal linearized ac models using incremental permeability data from a previous static solution, the use of nonlinear models must be restricted to the static limiting case of the low-frequency model.

To fully model nonlinearity in the rotor, a transient solution would be required. This would increase the computational cost of the solution; however, since a rotor-frame-of-reference model does not model the movement of the stator slots past the rotor but instead allows the current to transfer smoothly from one slot to the next, it should be possible to use a larger time step than would be required for a rotating machine model. If the field winding could be connected to an external circuit, this would allow saturated values to be obtained for the small-signal transient characteristics. However, while the commercial software used does support this type of circuit winding, it has been found that models with complex circuit windings usually fail. Although standard (racetrack or solenoid) windings are not a problem, a circuit winding consisting of arc conductors that represents the field winding of the generator causes the model to fail. The use of transient nonlinear models of the rotor should therefore be restricted to checking the validity of data obtained from linear models.

Both 2D and 3D methods have been used to estimate the machine characteristics for the design described above. Since the capabilities of the 2D and 3D modeling software used are significantly different, somewhat different approaches are required. Nevertheless, in both cases, the small-signal characteristics have been estimated, and no transient models have been used.

The 2D results were obtained from a set of three models: one for direct-axis MMF, one for quadrature-axis MMF, and one for field current. In each case, the model was solved for a number of frequencies using permeability data reloaded from a previous

Fig. 3. Flux distribution for a) direct b) quadrature axis.

static solution with full load current in the stator. It would have been possible to remove the need for one of these models by connecting an external circuit to the field winding, but the cost of solving additional 2D models is small, and the use of separate models allows the external impedance of the field circuit to be added later.

The 3D results were obtained using the same method, but using linear models of the rotor. An appropriate value of permeability for these models was chosen as $\iiint B \cdot B / \iiint B \cdot H$, where the integrals were evaluated in the appropriate parts of a nonlinear model with full load currents in the windings. The accuracy of this linear approximation can be checked by comparing the values of synchronous reactances obtained from the linear models with values obtained from the difference of two nonlinear solutions using

$$
L_{\text {diff }}=\frac{\Psi_{+\Delta}-\Psi_{-\Delta}}{\Delta i} .
$$

This is an established technique and is described in [5]. Estimates of the reactance from linear and nonlinear static models agree to within $6 \%$.

\section{RESULTS}

The values of the synchronous reactances obtained from the 2D and 3D models are listed in Table I (as "per unit" values). It is apparent that the quadrature-axis reactance is higher than the direct-axis reactance. This result is different from what is obtained when machines of classical design are considered, where the direct axis reactance is usually higher.

The flux plots in Fig. 3 illustrate the reason for this rather unusual proportion of values of $X_{d}$ and $X_{q}$. It is apparent that the quadrature-axis flux can flow for relatively long distances within a high-permeability material (diverter rings), whereas the direct-axis flux must repeatedly leave the iron and flow through the nonmagnetic regions occupied by the field winding. Note also that for a design without flux diverters (currently under consideration) this result may be different.

When the results from $2 \mathrm{D}$ and $3 \mathrm{D}$ modeling are compared there is a noticeable difference between the two sets, with the $2 \mathrm{D}$ results giving lower estimates of the synchronous reactances. 


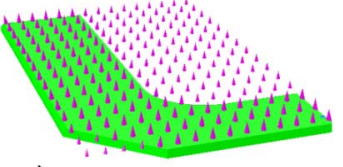

a)

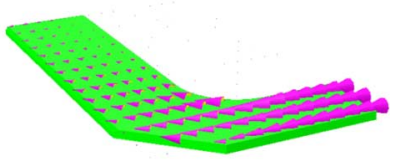

b)
Fig. 4. Flux distribution in one of the diverter rings a) direct b) quadrature flux distribution without field current.

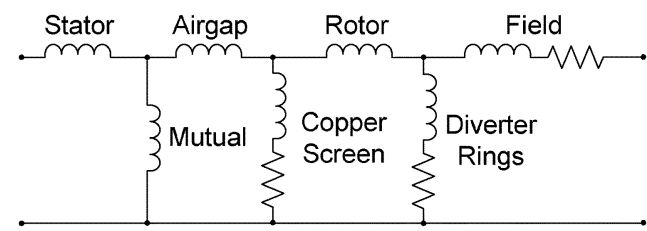

Fig. 5. Equivalent third-order circuit of the direct axis of the machine.

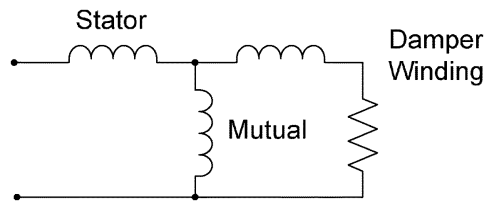

Fig. 6. Equivalent first-order circuit of the quadrature axis of the machine.

The results for the 2D estimation of both reactances include the end winding inductance which has been obtained by using the one coil model (without the conductivity in the copper screen) and expanding it to the mutual inductance matrix in the same manner as described in Section V. It has been found that its value is $0.150 \mathrm{mH}$. The higher values of reactance predicted using the 3D models can be attributed to the presence of additional flux paths that cannot be included in a $2 \mathrm{D}$ representation of the problem, in particular: the additional iron paths available to quadrature-axis flux in the ends of the diverter rings, and the large fringing flux at the core ends due to the large air gap and the extended rotor poles. The direct and quadrature flux paths in one of the rings are shown in Fig. 4.

The ac models were solved for the range of frequencies between 0.01 and $100 \mathrm{~Hz}$, and the flux linkage was extracted for each stator coil and for the rotor winding. As mentioned before, linear models were used and the field due to induced current in the field winding was added to the D-axis field of the stator winding by superposition. The resistance of the field circuit is not included in the FE models; a value of $10 \mathrm{~m} \Omega$ was assumed, which is an estimate of the resistance of the slip rings and current leads. Transfer functions corresponding to the equivalent circuits (Figs. 5 and 6) were fitted to the data from the ac models. The graphs (Figs. 7 and 8) suggest that these equivalent circuits are sufficient to model the response of the machine. These curves were obtained using the equivalent-circuit parameters shown in Table II. The base value of the field current was chosen arbitrarily as 16 A. Although this is only $10 \%$ of the rated field current, it represents substantially more MMF than full-load current in the stator. The use of significantly lower values does not permit the characteristics of the machine to be modeled by the equivalent circuit shown in Fig. 5 .

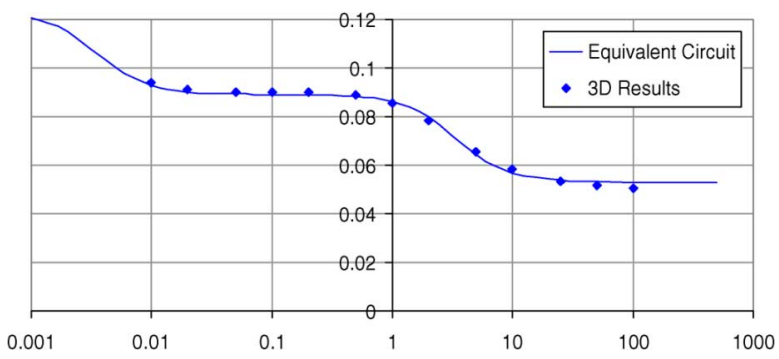

Fig. 7. Transfer function for the direct axis.

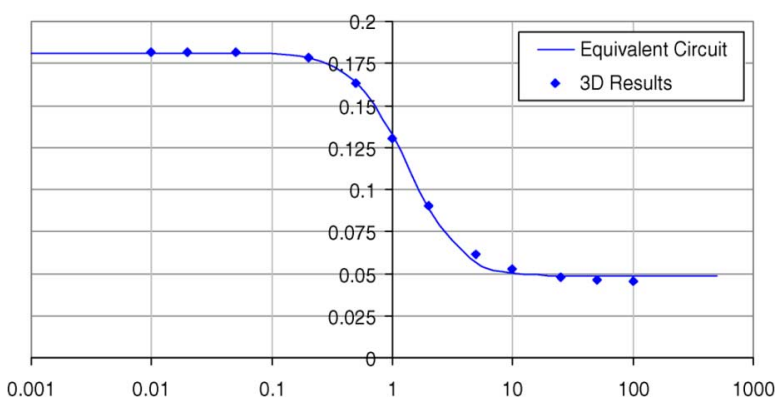

Fig. 8. Transfer function for the quadrature axis.

TABLE II

Parameters ObTained For The Circuits Elements [P.U]

\begin{tabular}{|c|c|c|c|c|c|c|c|}
\hline \multicolumn{8}{|c|}{ Direct axis } \\
\hline & Stator & Mutual & $\begin{array}{l}\text { Air } \\
\text { gap }\end{array}$ & $\begin{array}{c}\text { Copper } \\
\text { Screen } \\
\end{array}$ & Rotor & $\begin{array}{c}\text { Diverter } \\
\text { Rings }\end{array}$ & Field \\
\hline Reactance & 0.0136 & 0.1092 & 0.0306 & 0.0362 & 0.2086 & 0.0470 & 0.0059 \\
\hline Risistance & & & & 0.00982 & & 0.1366 & $2.56 \mathrm{e}-5$ \\
\hline \multicolumn{8}{|c|}{ Quadrature axis } \\
\hline Reactance & 0.017 & 0.164 & 0.039 & & & & \\
\hline Risistance & & & & 0.0054 & & & \\
\hline
\end{tabular}

\section{CONCLUSION}

A method of predicting the steady-state and transient behavior of a synchronous machine has been developed that does not require the use of transient finite-element models. Low-frequency ac models in the rotor frame of reference are used to model the field of the fundamental component of the stator MMF. Separate high-frequency models are used to predict the additional impedance due to MMF harmonics.

The method has been applied to a design with a coreless superconducting rotor, and it was shown that to represent this type of machine 3D models are required. The $\mathrm{D}$-axis equivalent circuit must be third-order since the diverter rings and copper screen act as separate damper windings.

\section{REFERENCES}

[1] M. K. Al-Mosawi, C. Beduz, and Y. Yang, "Construction of a 100 kVA high temperature superconducting synchronous generator," IEEE Trans. Applied Supercond., vol. 15, pp. 2182-2185, 2005.

[2] A. B. J. Reece and T. W. Preston, Finite Element Methods in Electrical Power Engineering. Oxford, U.K.: Oxford Univ. Press, 2000.

[3] "115-1995 IEEE Guide: Test Procedures for Synchronous Machines," 1995.

[4] I. M. Canay, "Determination of the model parameters of machines from the reactance operators $\mathrm{xd}(\mathrm{p}), \mathrm{xq}(\mathrm{p})$ (evaluation of standstill frequency response test)," IEEE Trans. Energy Conv., vol. 8, pp. 272-279, 1993.

[5] N. Bianchi, Electrical Machine Analysis Using Finite Elements. Boca Raton, FL: CRC, 2005. 